\title{
SUBSTRATE-INDUCED STRAIN IN EPITAXIAL LEAD CHALCOGENIDES BY GALVANOMAGNETIC EFFECT ROTATIONAL DEPENDENCE
}

\author{
N.N. Berchenko, O.A. Dobriansky, A.V. Korovin, A.Yu. Nikiforov, \\ V.S. YAKOVYNA
}

"Lviv Polytechnic" State University, 12 Bandera St, 290646 Lviv, Ukraine

AND H. ZogG

AFIF at Swiss Federal Institute of Technology, Technopark ETH-Teil 8005 Zürich, Switzerland

On the example of the $\mathrm{PbTe}$ and $\mathrm{Pb}_{0.77} \mathrm{Sn}_{0.23} \mathrm{Te}$ on $\mathrm{BaF}_{2}$ the possibility of using the weak magnetic field resistance technique for the evaluation of mismatch-thermally induced strains in semiconductors with multivalley band structure is discussed.

PACS numbers: $72.20 . \mathrm{My}, \mathbf{7 2 . 8 0 . J c}$

Substrate-induced strain in epitaxial thin films has long been recognized as a problem which impacts both fundamental studies and applications. Lead chalcogenides epitaxial films on $\mathrm{BaF}_{2}$ substrates due to their band structure specifics are a suitable object for investigation because in these semiconductors thermal expansion and lattice mismatched strains break the cubic structure of lattice and lead to a significant modification of the semiconductor band structure [1,2]. As it is shown by Allgaier [3], the distortion degree of band structure and accordingly the value of elastic strains for $n$-type $\mathrm{PbTe}$ films can be estimated using four-coefficient weak-field magnetoresistance measurement technique. We extended this technique for $p$-type material including samples protected with coatings.

Single-crystal films (up to $5 \mu \mathrm{m}$ thick) of $n$ - and $p$-type $\mathrm{PbTe}$ and $\mathrm{Pb}_{0.77} \mathrm{Sn}_{0.23} \mathrm{Te}\left(n, p=5 \times 10^{17} \mathrm{~cm}^{-3}, \mu_{n} \approx 3 \times 10^{4}\right.$ and $\mu_{p} \approx 1.2 \times 10^{4} \mathrm{~cm}^{2} \cdot \mathrm{V}^{-1} \cdot \mathrm{s}^{-1}$ at $77 \mathrm{~K}$ ) were prepared by the method of flash evaporation in vacuum on freshly cleaved (111) $\mathrm{BaF}_{2}$ substrates. To measure the Hall coefficient, electrical conductivity and magnetoresistance the standard Hall bar configuration was used. The samples were etched into their final shape by either photolithographic techniques or grown on $\mathrm{a} \mathrm{BaF}_{2}$ substrate through a bimetallic mask. To form the contact pads gold was deposited through masks. Ag wires were soldered to the pads 
(III)

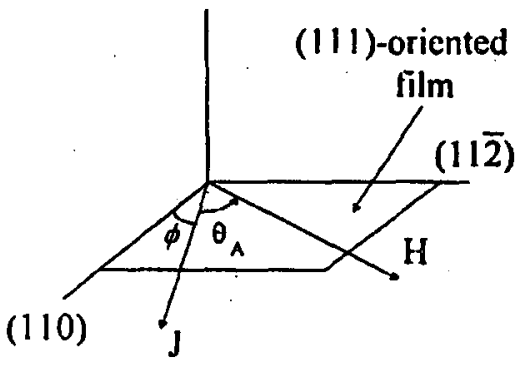

(111)

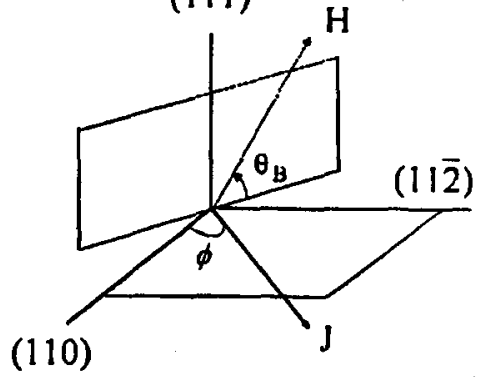

Fig. 1. Two configurations of magnetic field rotation. The angle $\phi$ is fixed by the photolithography or by mask, $\theta$ is the direction of the magnetic field. The sample current $I$ is always confined to the (111) plane. In configuration $A$ the magnetic field $H$ is also in (111) plane and in configuration $B$ the magnetic field is turned in plane normal to (111) plane, so $\theta_{B}$ is the angle between magnetic field and (111) plane.

with In. Measurements were conducted with a constant current $(50 \mu \mathrm{A})$ in a constant magnetic field satisfying the condition of weak magnetic field for fixed mobility of carriers. Rotational dependences were measured by means of rotation of the sample holder in magnetic field with the $5^{\circ}$ spacing. The two experimental configurations $A$ and $B$ (i.e. planes of rotation of the magnetic field used in the present study) are shown in Fig. 1. The magnitude of the weak-field magnetoresistance (WFMR) is examined in term of the dimensionless coefficients $M_{\phi}^{\theta}$

$$
\frac{\Delta \rho}{\rho_{0}}=M_{\phi}^{\theta}\left(\mu_{\mathbf{H}} B\right)^{2},
$$

where $\Delta \rho$ is the fractional change in zero-field resistivity $\rho_{0}, \mu_{\mathrm{H}}$ and $B$ are the magnitudes of the Hall mobility and magnetic field intensity,respectively. $M_{\phi}^{\theta}$ depends on the sample current and magnetic field directions $\phi$ and $\theta$ (Fig. 1). In a cubic crystal, particularly bulk PbTe, $M_{\phi}^{\theta}$ is completely described by the Seitz-Pearson-Sull coefficients $-b, c$, and $d$. As a result of strain, the symmetry of (111)-oriented film is reduced from cubic to trigonal. Only one additional parameter is needed to fully characterize the WFMR in such trigonal crystals $-d^{\prime}$. by

For two configurations used for rotational WFMR the coefficient $M_{\phi}^{\theta}$ is given

$$
\begin{aligned}
& M_{\phi}^{\theta_{A}}=b+\frac{1}{2} c+\frac{1}{3} d+\left(\frac{1}{2} c+\frac{1}{6} d\right) \cos \left(2 \theta_{A}\right), \\
& M_{\phi}^{\theta_{B}}=b+\frac{1}{4} d-\frac{1}{12} d \cos \left(2 \theta_{B}\right)+\frac{1}{6} \sqrt{2} d^{\prime} \cos (3 \phi) \sin \left(2 \theta_{B}\right) .
\end{aligned}
$$

Equations (2) and (3) are used to extract $b, c, d$, and $d^{\prime}$ from the measured WFMR. We take the value of the parameter $d^{\prime}$ to be indicative of the strain induced into the film. When $d=d^{\prime}$ the strain is absent and the cubic symmetry remains unchanged. 


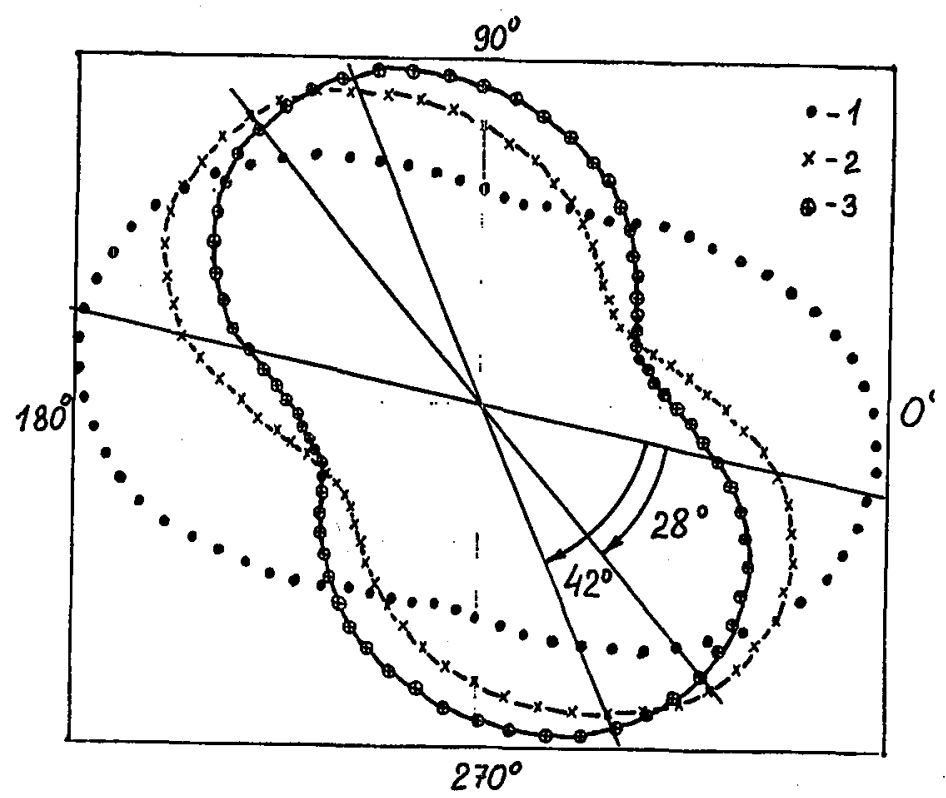

Fig. 2. Normalized $M_{\phi}^{\theta}$ versus $\theta_{B}$ in $B$-configuration for the epitaxial layers at $300 \mathrm{~K}$ (curve 1) and $77 \mathrm{~K}$ (curves 2 and 3 ) for the samples without $\mathrm{BaF}_{2}$ and with $\mathrm{BaF}_{2}$ evaporated film of $0.5 \mu \mathrm{m}$ thickness.

Experiments conducted on a great number of samples demonstrated that at room temperature the deformation is vanishingly small. But after cooling to $77 \mathrm{~K}$ a significant change in the form of the orientational dependences occurs, especially for the $B$ configuration (Fig. 2). The most visually impressive form of demonstrating this effect, a pronounced rotation of curve 2 about the initial figure (curve 1), is shown in Fig. 2. It is caused by the strain in the film. This increasing film distortion is ascribed to the thermal-expansion-coefficient difference between the semiconductor film and its $\mathrm{BaF}_{2}$ substrate at low temperature (Table).

\section{TABLE}

The main parameters of lead chalcogenides $\mathrm{BaF}_{2}$ and Si.

\begin{tabular}{l|c|c|c}
\hline \hline Material & $\begin{array}{c}\text { Lattice constant } \\
{[\mathrm{nm}]}\end{array}$ & \multicolumn{2}{|c}{$\begin{array}{c}\text { Thermal expansion } \\
\text { coefficient }\left[10^{-6} / \mathrm{K}\right]\end{array}$} \\
\cline { 3 - 4 } & & at $300 \mathrm{~K}$ & at $77 \mathrm{~K}$ \\
\hline $\mathrm{PbTe}$ & 0.646 & 19.8 & 17 \\
$\mathrm{PbSe}$ & 0.612 & 19.4 & - \\
$\mathrm{BaF}_{2}$ & 0.620 & 19.8 & 7 \\
$\mathrm{Si}$ & 0.543 & 2.6 & 0.6
\end{tabular}


The value $d^{\prime}$ has a significant scatter, but it may be, nevertheless, stated that the samples defined by photolithography possess a significantly lower value of the strain than the samples defined by the mask. Although after repeated cycles between room temperature and $77 \mathrm{~K}$ the value of $d^{\prime}$ in both cases is equally approaching $d$. The situation can be explained by extended defect creation [4] because it is accompanied by a charge carriers mobility decrease.

The potentialities of the weak-field magnetoresistance technique are well demonstrated by experiments performed for structures with $p-\mathrm{Pb}_{0.77} \mathrm{Sn}_{0.23} \mathrm{Te}$ epitaxial layer protected by anodic oxide (up to $200 \mathrm{~nm}$ thick) or by deposition of $\mathrm{BaF}_{2}$ (up to $0.5 \mu \mathrm{m}$ thick). All the measurements were conducted on the samples grown on the same piece of $\mathrm{BaF}_{2}$ substrate. These samples differed from each other only by the angle $\phi$. It has been found that while protecting coatings deposition the strain in epitaxial layer at $300 \mathrm{~K}$ is not changed within the precision of the experiment. At the same time, as it can be seen in Fig. 2, when for the sample without the $\mathrm{BaF}_{2}$ layer the angle of turning the rotational dependence $\Delta \rho$ in the $B$ configuration after cooling from 300 to $77 \mathrm{~K}$ is $28 \pm 5^{\circ}$ (curve 2), then for the sample covered with the $\mathrm{BaF}_{2}$ film the rotation angle value becomes as high as $42 \pm 5^{\circ}$ (curve 3). It indicates that the tensile strength increases. For anodic oxide the rotational dependences are not changed which is explained satisfactorily bythe glassy character of the anodic oxide. Strain relaxation in layers covered with $\mathrm{BaF}_{2}$ occurs after first few thermal cycles in contrast to the more slower relaxation of the initial samples.

As it has been demonstrated by Zogg et al. [5], low-cost IR sensors may be fabricated on the basis of lead chalcogenide epitaxial layers on Si (111). Table shows that in this case thermal mismatch strains are even more stronger. Thus, it is of a great interest to apply the WFMR technique to study these structures.

\section{References}

[1] J.R. Burke, G.R. Carver, Phys. Rev. B 17, 2719 (1979).

[2] D.V. Shamshur, R.V. Parfen'ev, D.V. Mashovets, A.V. Matveenko, V.V. Kosarev, K.I. Geiman, Sov. Phys. Semicond. 16, 798 (1982).

[3] R.S. Allgaier, Phys. Rev. B 31, 3822 (1985).

[4] J.B. Restorff, R.S. Allgaier, B. Houston, J. Appl. Phys. 52, 6185 (1981).

[5] H. Zogg, A. Fach, J.John, J. Masek, P. Muller, C. Paglino, S. Blunier, Opt. Eng. 34, 1964 (1995). 